\title{
Sazonalidade e Padrões Diários de Atividade de Machos de Euglossina (Hymenoptera: Apidae: Apini) e Preferências por Fragrâncias Artificiais em um Remanescente de Brejo de Altitude na Paraíba
}

\author{
Rodrigo Cesar Azevedo Pereira Farias ${ }^{\bowtie}$ \& Celso Feitosa Martins
}

Universidade Federal da Paraíba, e-mail: rodrigoento@gmail.com (Autor para correspondência ${ }^{\bowtie}$ ), cmartins@dse.ufpb.br.

\author{
EntomoBrasilis 6 (3): 202-209 (2013)
}

Resumo. As abelhas Euglossina ocorrem exclusivamente na região neotropical, com cerca de 200 espécies descritas. Utilizando-se fragrâncias artificiais como iscas, é possível realizar levantamentos faunísticos e estudar diversos aspectos ecológicos do grupo. Com o intuito de contribuir ao conhecimento da biodiversidade dos brejos de altitude, no tocante às abelhas Euglossina, sete fragrâncias artificiais foram utilizadas como iscas em um remanescente na cidade de Areia, Paraíba, em distintos períodos climáticos. Foram coletados 2.981 machos pertencentes a 14 espécies de Euglossina. A abundância e a riqueza foram maiores no período de estiagem, havendo variação no horário de atividade de um período para o outro. De modo geral, no período chuvoso os machos foram mais ativos após as 11h e, no de estiagem, entre 8h e 11h. Euglossa carolina (Nemésio), Eulaema nigrita (Lepeletier) e Eulaema cingulata (Fabricius) foram as espécies mais abundantes nos dois períodos. Beta ionona foi a fragrância que atraiu o maior número de espécies e indivíduos.

Palavras-chave: Abelhas das orquídeas; Euglossa; Eulaema; Exaerete; Mata do Pau-Ferro.

\section{Seasonality and Daily Activity Patterns of Euglossina males (Hymenoptera: Apidae: Apini) and Preferences for Artificial Fragrances in a Montane Atlantic Forest Remnant, Paraíba}

Abstract. Euglossina bees are distributed exclusively in the Neotropical Region, with around 200 species described. By the usage of artificial fragrances as baits, it is possible to accomplish faunistic inventories and study several ecological aspects of the group. To contribute to the knowledge of the biodiversity of a Montane Atlantic Forest remnant, concerning to Euglossina, seven fragrances were used as baits in different climatic periods. A total of 2,981 males belonging to 14 species of Euglossina were collected. Abundance and richness were higher in the dry period. Changes in the daily activity patterns were observed from a period to the other. Generally, in the rainy season the males were more active after 11am and in the dry season between 8am and 11am. Euglossa carolina (Nemésio), Eulaema nigrita (Lepeletier) and Eulaema cingulata (Fabricius) were the most abundant species on both periods. Ionone beta was the fragrance which attracted more species and specimens.

Keywords: Euglossa; Eulaema; Exaerete; Mata do Pau-Ferro; Orchid bees.

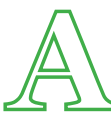
s abelhas da subtribo Euglossina (sensu Silveira et al. 2002) formam um grupo de insetos geralmente de coloração metálica, amplamente distribuídos na região neotropical, apresentando comportamento que varia do nível solitário até social (Dodson et al. 1969; DressLER 1982; Michener 2000; Garófalo 2006), com cerca de 200 espécies descritas (Moure et al. 2007). São encontrados na faixa compreendida entre $30^{\circ}$ de latitude norte e $32^{\circ}$ de latitude sul, mas algumas espécies ocorrem além dos trópicos (REBÊLO 2001). A diversidade é maior em florestas úmidas, com poucas espécies ocorrendo em savanas e florestas de galeria (DRESSLER 1982; RouBiK \& HANSON 2004). As Euglossina compreendem cerca de $25 \%$ de todas as espécies de abelhas que residem em qualquer floresta de baixa a média elevação nos trópicos úmidos americanos (Roubik \& HANSON 2004).

Os machos das espécies de Euglossina apresentam comportamento característico de coletar substâncias aromáticas em plantas, dentre as quais as orquídeas se destacam com centenas de espécies visitadas, mas também visitam espécies de Araceae, Gesneriaceae, Solanaceae e Euphorbiaceae, entre outras (ACKeRMAN 1983b; Williams \& WhitTEN 1983).
O maior passo para o conhecimento mais detalhado das Euglossina ocorreu quando se descobriu que os machos eram atraídos por substâncias aromáticas, análogas àquelas presentes nas fragrâncias das orquídeas. A utilização de iscas odoríferas para atrair machos de Euglossina, por ser um método prático e eficiente que permite realizar rapidamente uma amostragem da comunidade dessas abelhas numa determinada área, possibilita a coleta de dados básicos referentes a sua biologia, como riqueza de espécies, abundância, horário de atividade e preferência por fragrâncias.

Há dezenas de estudos sobre a composição de Euglossina em fragmentos de mata atlântica no Brasil (Mattozo et al. 2011; Aguiar \& Gaglianone 2012), mas este é o primeiro realizado em um remanescente de Brejo de Altitude, ambiente peculiar da mata atlântica nordestina, considerando os padrões diários de atividade dos machos atraídos às iscas odoríferas em distintos períodos climáticos.

Agência de financiamento: Coordenação de Aperfeiçoamento de Pessoal de Nível Superior (CAPES) - bolsa do primeiro autor 


\section{MATERIAL E MÉTODOS}

Área de estudo. O CONAMA (1993) definiu brejo de altitude como "mancha de floresta que ocorre no nordeste do país, em elevações e platôs onde ventos úmidos condensam o excesso de vapor e criam um ambiente de maior umidade". Os brejos de altitude são reconhecidos como ecossistemas associados à mata atlântica (Floresta Estacional Semidecidual Montana), cuja característica principal é a presença da mata úmida, além de apresentar clima úmido e com temperaturas amenas, altitude que pode ultrapassar $700 \mathrm{~m}$ e maior diversidade de vegetação, caracterizada por florestas semidecíduas e ombrófilas abertas. Essas características fazem com que os animais da região semiárida, no entorno dos brejos, procurem refúgio nas serras durante os grandes períodos de estiagem (РôRTo et al. 2004).

De acordo com VASConcelos-Sobrinho (1971) existem 43 brejos na floresta atlântica nordestina, distribuídos nos estados do Ceará, Rio Grande do Norte, Paraíba e Pernambuco. Deste total, 31 estão localizados em Pernambuco e na Paraíba. Atualmente, restam 2.626,68 $\mathrm{Km}^{2}(14,2 \%)$ da vegetação original dos brejos. $\mathrm{O}$ valor da vegetação remanescente torna os brejos o setor mais ameaçado da floresta atlântica brasileira (TABARELli \& SANTOS 2004). Na avaliação e identificação de áreas e ações prioritárias para conservação, utilização sustentável e repartição de benefícios da biodiversidade brasileira, o MMA (2002) chamou a atenção para os brejos do nordeste, tendo em vista o grande potencial para ocorrência de endemismos e espécies ainda desconhecidas.

A Reserva Ecológica da Mata do Pau-Ferro está localizada a 5 $\mathrm{km}$ da sede do município de Areia, PB (6 ${ }^{\circ} 58^{\prime} 12^{\prime}$ S S e $35^{\circ} 42^{\prime} 15^{\prime}$ W), e, devido ao seu tamanho e estado de conservação, é a mata de brejo mais representativa no estado da Paraíba (BArbosa et al. 2004). A altitude da região varia entre 400 e $600 \mathrm{~m}$, com temperatura média anual de $22^{\circ} \mathrm{C}$, umidade relativa em torno de $85 \%$ e os totais pluviométricos anuais em torno de $1.400 \mathrm{~mm}$. A Mata do Pau-Ferro foi classificada como Floresta Estacional Subperenifólia de Altitude (Mayo \& Fevereiro 1982). A área da reserva compreende 600 ha de mata, com córregos, trilhas e uma barragem. Segundo o MMA (2002), é uma das áreas prioritárias para conservação da flora, anfíbios, répteis e aves representativos dos brejos de altitude. Entretanto, no tocante a invertebrados, a área é tratada como "insuficientemente conhecida, mas de provável importância biológica”.

Amostragem. Foram utilizadas sete fragrâncias artificiais para atrair os machos de Euglossina: acetato de benzila, beta ionona, escatol, eucaliptol, eugenol, salicilato de metila e vanilina. O modelo de iscas e os procedimentos de captura das abelhas foram os mesmos descritos por FARIAS et al. (2007, 2008).

Foram realizadas 12 atividades diárias de campo, sendo seis no período chuvoso (de 26 de julho a $1^{\circ}$ de agosto de 2005) e seis no período de estiagem (de 17 a 22 de janeiro de 2006), sempre das 07 hoomin às 15 hoomin. Os períodos climáticos selecionados foram baseados nas médias históricas de climatologia para a cidade de Areia (Figura 1). As atividades do dia 30 de julho de 2005 foram canceladas devido à chuva que perdurou o dia inteiro.

Com base em um estudo piloto realizado em março de 2005, seis pontos de coleta foram definidos na mata ao longo de um transecto de cerca de $1.500 \mathrm{~m}$, o qual se iniciava a $500 \mathrm{~m}$ da borda, seguindo a trilha principal que corta a mata. Cada ponto distava aproximadamente $250 \mathrm{~m}$ entre si e em cada dia era sorteado o ponto de coleta. As iscas eram reabastecidas a cada quatro horas.

Em laboratório, as abelhas capturadas foram montadas, colocadas em estufa por 24 horas e etiquetadas, conforme método padrão de preparação de coleções entomológicas (Almeida et al. 2012) e depositados na Coleção Entomológica do Departamento de Sistemática e Ecologia da Universidade Federal da Paraíba.

Análises dos dados. Para cada espécime, as informações sobre o nome da espécie, local de coleta, fragrância em que foi amostrado, horário, data, temperatura e umidade foram incluídas em um banco de dados do programa Microsoft Excel 2007®. Para análise de variância na distribuição dos dados de abundância dos machos coletados por horário foi calculada a ANOVA. As análises de correlação entre o número de indivíduos e os valores médios de temperatura e umidade, bem como a relação entre temperatura e umidade, foram feitas empregando-se o coeficiente de correlação paramétrico de Pearson (r). Estas análises foram feitas através do programa estatístico BioEstat versão 5.0@.

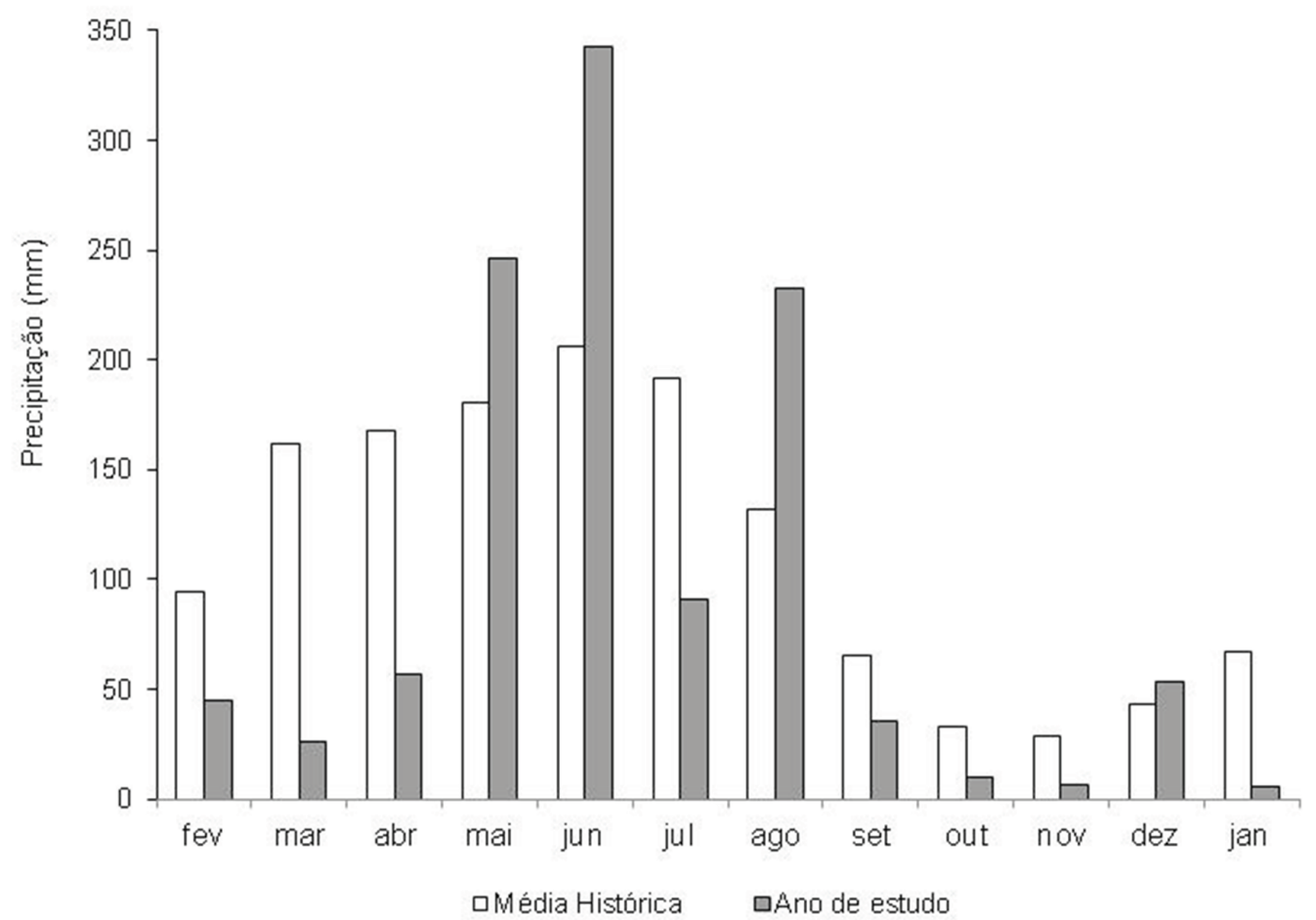

Figura 1. Valores totais de precipitação mensal de fevereiro de 2005 a janeiro de 2006 na cidade de Areia, PB, associados aos valores históricos (Fonte: AESA - Agência Executiva de Gestão das Águas do Estado da Paraíba). 


\section{RESULTADOS}

Foram capturados 2.981 machos pertencentes a 14 espécies de Euglossina (Tabela 1). Euglossa carolina (Nemésio) foi a espécie queapresentou maiorabundância: 1.888 , correspondendoa $63,4 \%$ de todos os indivíduos, seguida por Eulaema nigrita (Lepeletier), Eulaema cingulata (Fabricius), Euglossa nanomelanotricha (Nemésio), Euglossa fimbriata (Rebêlo \& Moure) e Euglossa perpulchra (Moure \& Schlindwein) (Tabela 1). Em conjunto, essas espécies representaram 99,6\% dos indivíduos amostrados.

Do número total de indivíduos, 325 foram registrados no período chuvoso e 2.656 no período de estiagem. Das espécies amostradas, oito estiveram presentes nas duas estações e seis só ocorreram no período de estiagem. As espécies coletadas somente no período de estiagem foram representadas por um ou dois indivíduos. Euglossa violaceifrons Rebêlo \& Moure teve um indivíduo coletado em cada período. As espécies E. carolina, $E$. cingulata e $E$. nigrita foram mais abundantes em ambos os períodos (Tabela 1).

Houve variação nos horários de maior atividade dos machos entre os períodos chuvosos $(\mathrm{F}=3,01 ; \mathrm{p}=0,012 ; \mathrm{N}=48)$ e de estiagem $(\mathrm{F}=7,50 ; \mathrm{p}=0,0001 ; \mathrm{N}=48)$ (Tabela 2 e Tabela 3). No período chuvoso, de modo geral, os machos foram mais ativos após as $11 \mathrm{~h}$ e, no de estiagem, entre $8 \mathrm{~h}$ e $11 \mathrm{~h}$.

O teste de Pearson mostrou forte correlação positiva $(\mathrm{r}=0,87$; $\mathrm{p}=0,004$ ) entre o número de indivíduos e a temperatura no período chuvoso, mostrando que nesse período quanto maior a temperatura, maior é o número de indivíduos nas iscas. Também no mesmo período, houve uma forte correlação negativa $(\mathrm{r}=-0,91$; $\mathrm{p}=\mathrm{o}, \mathrm{o01}$ ) entre o número de indivíduos e a umidade relativa. Assim sendo, altos valores de umidade relativa influenciaram negativamente o número de indivíduos coletados.

No período de estiagem, não houve correlações entre o número de indivíduos e os valores médios de temperatura e umidade.

Todas as fragrâncias utilizadas foram atrativas nos dois períodos. Beta ionona e eucaliptol foram as que atraíram o maior número de espécies (Tabela 4).

As espécies com mais de um indivíduo amostrado na Mata do PauFerro foram coletadas em mais de uma fragrância. Entretanto, algumas demonstraram preferência por uma ou duas fragrâncias em particular. Entre as espécies mais abundantes, os machos de $E$. carolina foram capturados em todas as fragrâncias, porém $74,6 \%$ na beta ionona; $E$. cingulata em todas as fragrâncias, sendo $43 \%$ no acetato de benzila; e $E$. nigrita em cinco fragrâncias, sendo $82,8 \%$ no escatol (Tabela 4 ).

Observando-se o número de indivíduos atraídos às fragrâncias nos dois períodos, percebe-se que a beta ionona, o escatol e o eucaliptol foram sempre mais atrativos, o que foi determinado pela atratividade às espécies mais abundantes. Não foram observadas diferenças no número proporcional de visitas às iscas entre ambos os períodos de estudo, evidenciando que as fragrâncias utilizadas exerceram o mesmo poder relativo de atração.

Tabela 1. Abundância total (N), abundância (n) e frequência (\%) relativas e número de espécies de Euglossina (S) registradas na Reserva Ecológica da Mata do Pau-Ferro, Areia, PB, nos períodos chuvoso (26/07 a 01/08/05) e de estiagem (17 a 22/01/06).

\begin{tabular}{|c|c|c|c|c|}
\hline \multirow{3}{*}{ Espécies } & \multicolumn{2}{|c|}{$\mathbf{N}$} & \multirow{3}{*}{ Total } & \multirow{3}{*}{$\%$} \\
\hline & \multicolumn{2}{|c|}{ Período } & & \\
\hline & chuvoso & estiagem & & \\
\hline Euglossa carolina (Nemésio) & 190 & 1.698 & 1.888 & 63,4 \\
\hline Eulaema nigrita (Lepeletier) & 89 & 603 & 692 & 23,3 \\
\hline Eulaema cingulata (Fabricius) & 30 & 200 & 230 & 7,7 \\
\hline Euglossa nanomelanotricha (Nemésio) & 5 & 75 & 80 & 2,7 \\
\hline Euglossa fimbriata (Rebêlo \& Moure) & 1 & 42 & 43 & 1,4 \\
\hline Euglossa perpulchra Moure \& Schlindwein & 8 & 24 & 32 & 1,1 \\
\hline Eulaema bombiformis (Packard) & 1 & 6 & 7 & 0,2 \\
\hline Euglossa securigera Dressler & o & 2 & 2 & 0,1 \\
\hline Euglossa violaceifrons Rebêlo \& Moure & 1 & 1 & 2 & 0,1 \\
\hline Euglossa townsendi Cockerell & o & 1 & 1 & 0,0 \\
\hline Euglossa sp. & o & 1 & 1 & 0,0 \\
\hline Eulaema atleticana Nemésio & o & 1 & 1 & 0,0 \\
\hline Exaerete frontalis (Guérin) & o & 1 & 1 & 0,0 \\
\hline Exaerete smaragdina (Guérin) & o & 1 & 1 & 0,0 \\
\hline $\mathbf{n}$ & 325 & 2.656 & 2.981 & 100 \\
\hline $\mathbf{S}$ & 8 & 14 & 14 & - \\
\hline
\end{tabular}

Tabela 2. Abundância (n) e frequência (\%) relativas de machos de Euglossina atraídos às iscas odoríferas, relacionados às médias de temperatura (T) e umidade relativa (UR) nos diferentes intervalos de horas na Mata do Pau-Ferro, Areia, PB, no período chuvoso (26/07 a 01/08/05).

\begin{tabular}{ccccc}
\hline \multirow{2}{*}{ Horário } & \multirow{2}{*}{$\mathbf{n}$} & $\mathbf{\%}$ & \multicolumn{2}{c}{ Valores médios } \\
\cline { 4 - 5 } & & & $\mathbf{T}\left({ }^{\circ} \mathbf{C}\right)$ & UR (\%) \\
\hline 7:00 - 8:00 & 8 & 2,46 & 20,4 & 88,4 \\
8:00 - 9:00 & 29 & 8,92 & 20,9 & 84,4 \\
\hline 9:00-10:00 & 43 & 13,23 & 21,9 & 79,2 \\
\hline 10:00-11:00 & 37 & 11,38 & 23,3 & 72,9 \\
\hline 11:00 - 12:00 & 45 & 13,85 & 23,8 & 72,9 \\
\hline 12:00 - 13:00 & 61 & 18,77 & 24 & 70,1 \\
\hline 13:00 - 14:00 & 55 & 16,92 & 23,7 & 70,2 \\
\hline 14:00 - 15:00 & 47 & 14,46 & 23,3 & 73,7 \\
\hline
\end{tabular}

Tabela 3. Abundância (n) e frequência (\%) relativas de machos de Euglossina atraídos às iscas odoríferas, relacionados às médias de temperatura (T) e umidade relativa (UR) nos diferentes intervalos de horas na Mata do Pau-Ferro, Areia, PB, no período de estiagem (17 a 22/01/06)

\begin{tabular}{ccccc}
\hline \multirow{2}{*}{ Horário } & \multirow{2}{*}{$\mathbf{n}$} & $\mathbf{\%}$ & \multicolumn{2}{c}{ Valores médios } \\
\cline { 4 - 5 } & & & $\mathbf{T}\left({ }^{\circ} \mathbf{C}\right)$ & UR (\%) \\
\hline 7:00 - 8:00 & 254 & 9,56 & 24,4 & 77,8 \\
\hline 8:00 - 9:00 & 407 & 15,32 & 25,9 & 68,3 \\
\hline 9:00 - 10:00 & 472 & 17,76 & 27,7 & 60,6 \\
\hline 10:00 - 11:00 & 479 & 18,03 & 29,3 & 52,4 \\
\hline 11:00 - 12:00 & 339 & 12,76 & 30,8 & 45,5 \\
\hline 12:00-13:00 & 275 & 10,35 & 31,5 & 41,9 \\
\hline 13:00 - 14:00 & 238 & 8,96 & 31,3 & 41,7 \\
\hline 14:00 - 15:00 & 193 & 7,26 & 30,8 & 44,3 \\
\hline
\end{tabular}


Tabela 4. Abundância (n) e frequência (\%) relativas e número de espécies (S) de Euglossina, em relação às fragrâncias utilizadas, na Reserva Ecológica da Mata do Pau-Ferro, Areia, PB, nos períodos chuvoso (26/07 a 01/08/05) e de estiagem (17 a 22/01/06).

\begin{tabular}{|c|c|c|c|c|c|c|c|c|c|c|c|c|c|c|}
\hline \multirow{3}{*}{ Espécie } & \multicolumn{14}{|c|}{ Fragrâncias* visitadas } \\
\hline & \multicolumn{2}{|c|}{ ACB } & \multicolumn{2}{|c|}{ BIO } & \multicolumn{2}{|c|}{ ESC } & \multicolumn{2}{|c|}{ EUC } & \multicolumn{2}{|c|}{ EUG } & \multicolumn{2}{|c|}{ SLM } & \multicolumn{2}{|c|}{ VAN } \\
\hline & $\mathbf{n}$ & $\%$ & $\mathbf{n}$ & $\%$ & $\mathbf{n}$ & $\%$ & $\mathbf{n}$ & $\%$ & $\mathbf{n}$ & $\%$ & $\mathbf{n}$ & $\%$ & $\mathbf{n}$ & $\%$ \\
\hline Euglossa carolina (Nemésio) & 3 & 0,2 & 1.408 & 74,5 & 26 & 1,4 & 435 & 23 & 1 & 0,1 & 8 & 0,4 & 7 & 0,4 \\
\hline Eulaema nigrita (Lepeletier) & & & 7 & 16,3 & 1 & 2,3 & 28 & 65,1 & 7 & 16,3 & & & & \\
\hline Eulaema cingulata (Fabricius) & & & 2 & 2,5 & & & 75 & 93,7 & 3 & 3,8 & & & & \\
\hline Euglossa nanomelanotricha (Nemésio) & 1 & 3,1 & 12 & 37,5 & & & 2 & 6,3 & & & & & 17 & 53,1 \\
\hline Euglossa fimbriata (Rebêlo \& Moure) & & & 1 & 50 & & & & & 1 & 50 & & & & \\
\hline Euglossa perpulchra Moure \& Schlindwein & & & 1 & 100 & & & & & & & & & & \\
\hline Eulaema bombiformis (Packard) & & & 1 & 50 & & & & & 1 & 50 & & & & \\
\hline Euglossa securigera Dressler & & & & & 1 & 50 & 1 & 50 & & & & & & \\
\hline Euglossa violaceifrons Rebêlo \& Moure & 5 & 71,4 & & & & & & & & & 2 & 28,6 & & \\
\hline Euglossa townsendi Cockerell & 99 & 43 & 6 & 2,6 & 59 & 25,7 & 5 & 2,2 & 21 & 9,1 & 1 & 0,4 & 39 & 17 \\
\hline Euglossa sp. & 1 & 100 & & & & & & & & & & & & \\
\hline Eulaema atleticana Nemésio & 7 & 1 & 12 & 1,7 & 573 & 82,8 & 35 & 5,1 & 2 & 0,3 & & & 63 & 9,1 \\
\hline Exaerete frontalis (Guérin) & & & & & 1 & 100 & & & & & & & & \\
\hline Exaerete smaragdina (Guérin) & & & & & & & 1 & 100 & & & & & & \\
\hline $\mathbf{n}$ & 116 & 3,9 & 1.450 & 48,6 & 661 & 22,2 & 582 & 19,5 & 36 & 1,2 & 11 & $\mathbf{0 , 4}$ & 126 & 4,2 \\
\hline $\mathbf{S}$ & & & & & & & & & & & & & & \\
\hline
\end{tabular}

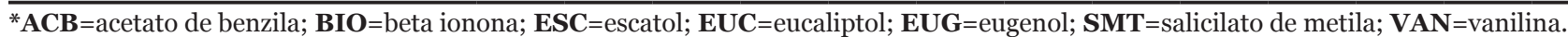

\section{DISCUSSÃO}

Segundo Roubik (2001) e (Ackerman 1983a), é de se esperar que haja variação sazonal na abundância de Euglossina, pois as populações de algumas espécies podem permanecer ativas, porém reduzidas em tamanho durante certos períodos do ano. Até mesmo nos trópicos as comunidades de Euglossina se modificam durante o ano. Enquanto a maioria está presente como adulto ao longo do ano, elas são dinâmicas e têm picos distintos e baixas estações (Roubik \& Hanson 2004). Por esta razão que, mesmo no norte e nordeste do Brasil, onde não há quatro estações definidas ao longo do ano, mas períodos de chuva e de estiagem, tem-se observado variação sazonal na riqueza e abundância das abelhas Euglossina.

Diversos autores (BECKER et al. 1991; RebêLo \& GARÓFALO 1991; Silva \& Rebêlo 1999; Brito \& RÊGo 2001; Silva \& RebÊlo 2002; VIANA et al. 2002) verificaram que as espécies e os indivíduos foram mais abundantes no período mais úmido. REBÊLO \& CABRAL (1997) obtiveram dados semelhantes e ainda observaram que no mesmo período foram registradas as menores médias de temperatura. NEVES \& VIANA (1997) registraram maior atividade nos meses mais quentes e com as médias de temperatura em torno dos $30^{\circ} \mathrm{C}$. Na área estudada por Neves \& Viana (1999), os machos foram mais abundantes nos meses mais secos do ano. BEZERRA \& MARTins (2001), por sua vez, observaram que os machos foram mais abundantes do meio do período de estiagem ao início do chuvoso, e Oliveira (1999) obteve maior abundância e riqueza do fim do período de estiagem para o início do período chuvoso.

Alguns autores têm sugerido que flutuações sazonais nas abundâncias das espécies são resultantes das atividades de nidificação e padrões de emergências das abelhas na área e não da imigração a partir de outros hábitats (ACKERMAN 1983a; RoUBIK \& ACKERMAN 1987; REBÊLO \& GARÓFALO 1991), como sugerido por JANZEN et al. (1982). ViANA et al. (2002), por sua vez, sugeriram que os dois fatores possam estar envolvidos, pois a área onde esses autores realizaram seu estudo é um mosaico de ecossistemas como matas, dunas cobertas por vegetação de restinga e brejos, onde a distribuição de recursos não é homogênea nem mesmo ao longo do dia. Com relação à Mata do Pau-Ferro, é necessário que se façam levantamentos nas áreas de caatinga adjacentes para verificar se a maior abundância de Euglossina no período seco é um reflexo da dispersão dos machos vindos da caatinga como consequência da escassez de recursos nesse período [pois é sabido que os animais da região semiárida no entorno dos brejos procuram refúgio nas serras durante os grandes períodos de estiagem (CAvalcantr 2004)]. Por outro lado, pode ser simplesmente que as atividades de nidificação e padrões de emergência locais sejam os responsáveis por tal variação, como ACKERMAN (1983a) argumentou.

O horário de atividade dos machos de Euglossina tem se mostrado bastante similar nos diversos estudos realizados, com o pico de atividade geralmente entre 7 h e 12h. Neves \& Viana (1999); Bezerra \& Martins (2001); Silva \& RebêLo (2002) e Viana et al. (2002) observaram maior atividade entre 8h e 1oh. BRAGA (1976) observou maior atividade entre $8 \mathrm{~h}$ e 11h. BRITO \& RÊGO (2001) observaram maior atividade dos machos entre gh e 1oh. Powell \& Powell (1987) e Silva \& RebêLo (1999) observaram maior abundância entre $9 \mathrm{~h}$ e 12h. CARVALHO et al. (2006) registraram alta atividade dos machos em todos os horários entre $8 \mathrm{~h}$ e $12 \mathrm{~h}$, com notável diminuição após o meio-dia. No Panamá, DoDson et al. (1969) observaram que os machos são mais ativos entre 7 h e 13h. Por outro lado, Oliveira (1999) observou picos de atividade dos machos entre $12 \mathrm{~h}$ e $14 \mathrm{~h}$.

Segundo Roubik \& Hanson (2004), a maioria das espécies voa quando condições gerais são boas, normalmente quando o tempo está quente e relativamente úmido. DrEssLer (1982) explicou que a atividade de coleta de fragrâncias é bem maior pela manhã, mas esporadicamente pode se estender pela tarde.

BRAGA (1976) argumentou que a diferença de temperatura influencia na atividade das Euglossina. Ambruster \& Berg (1994), numa floresta de terra firme na Venezuela, fizeram intensas observações sobre a relação da atividade dos machos e a temperatura do ambiente. Os autores constataram que a atividade dos machos de Euglossina nas iscas reflete seus padrões naturais de atividade. $O$ efeito térmico do ambiente tem muito mais influência na atividade das abelhas do que os efeitos da dispersão e volatilização das fragrâncias, os quais podem ser positivamente influenciados pela velocidade do vento e por temperaturas mais elevadas, que ocasionam o aumento do tamanho das plumas de odor. Todavia, segundo os autores, tais efeitos não são tão importantes quanto os efeitos do clima sobre as abelhas. AcKerman (1983a) mostrou claramente que a atividade das abelhas independe do vento, sendo a dinâmica 
das plumas de odor, portanto, menos importante do que outros fatores. Ambruster \& Berg (1994) ainda constataram que as fragrâncias mais visitadas (eucaliptol e salicilato de metila) são extremamente voláteis mesmo sob baixas temperaturas e isso não interferiu no horário de visitas às iscas.

Santos \& Sofia (2002) estudaram o horário de atividade dos machos de Euglossina no Paraná e verificaram que no período quente e chuvoso a atividade foi maior entre $10 \mathrm{~h}$ e $11 \mathrm{~h}$ (com temperaturas médias variando entre $22,2^{\circ}$ a $26,5^{\circ} \mathrm{C}$ ), enquanto que período seco e frio os machos foram mais ativos entre $11 \mathrm{~h} \mathrm{e}$ $14 \mathrm{~h}$ (com temperaturas médias variando entre $21,2^{\circ}$ e $23,3^{\circ} \mathrm{C}$ ). $\mathrm{Na}$ Mata do Pau-Ferro, durante o período chuvoso, as temperaturas médias variaram entre $19,7^{\circ}$ e $25,5^{\circ} \mathrm{C}$ ao longo dia e entre $21,5^{\circ} \mathrm{e}$ $25,3^{\circ} \mathrm{C}$ nos horários de maior atividade. No período de estiagem, as médias de temperatura variaram entre $22,5^{\circ}$ e $32,6^{\circ} \mathrm{C}$ ao longo do dia e entre $24,5^{\circ}$ e $29,8^{\circ}$ nos horários de maior atividade.

TonHASCA et al. (2002a) não obtiveram correlação entre a abundância dos machos e a temperatura ou umidade relativa, mas observaram que a abundância das abelhas foi sempre menor quando a temperatura estava em torno de $22^{\circ} \mathrm{C}$. Porém, é certo que a maior atividade dos machos sob temperaturas mais elevadas atinge um ápice e depois começa a decair, caso contrário os machos podem sofrer superaquecimento (AMBRUSTER \& BERG 1994). BRAGA (1976) verificou que quando se aproxima o meiodia ocorre uma queda brusca nas atrações e que os machos só visitam as áreas mais abertas nas horas menos quentes do dia. TonHASCA et al. (2002b) relataram que a atividade de voo das euglossinas reduz acentuadamente quando a temperatura atinge $30^{\circ} \mathrm{C}$. Segundo Ambruster \& Berg (1994), a temperatura é crucial para determinar o início da atividade dos machos num determinado dia, o que pode coincidir com os diversos picos de atividade observados nos trabalhos, considerando que a grande parte dos machos estará ativa naquelas horas. Os autores ainda argumentam que nas primeiras horas da manhã os machos de Euglossina tendem a permanecer inativos até que a temperatura do ambiente atinja certo grau (aproximadamente $23^{\circ} \mathrm{C}$ ). A partir daí, durante o resto do dia, o forrageio em busca de fragrâncias parece ser pouco influenciado pela temperatura do ambiente e os machos são ativos segundo seus ritmos circadianos.

INOUYE (1975) também postulou que a temperatura é um fator determinante na atividade dos machos. O autor estudou as temperaturas corpóreas de machos de nove espécies de Euglossa durante o voo e observou médias de temperatura variando de $37,4^{\circ}$ a $40,2^{\circ} \mathrm{C}$. Como as euglossinas não possuem um mecanismo tão eficaz de manutenção da temperatura corpórea, acredita-se, que quando a atividade nas iscas odoríferas diminui, os machos não cessam o forrageio por néctar. Segundo o autor, as fragrâncias não são uma recompensa calórica, portanto a manutenção de altas temperaturas corpóreas em relação à temperatura do ambiente pode não ser compatível com os gastos energéticos das abelhas, diferentemente do forrageio por néctar, que é uma tarefa metabolicamente recompensadora. Esta seria a razão pela qual os machos visitam muito pouco as iscas nas horas mais quentes. Se a observação dos machos em flores durante a coleta de néctar fosse uma situação comum, seria fácil comprovar ou não a hipótese do autor. Segundo Oliveira (1999), ainda não são claros os fatores que poderiam estar determinando a atividade das Euglossina, mas após discutir as várias explicações apresentadas em outros estudos, o autor está certo de que não apenas um, mas vários fatores abióticos e bióticos têm influência no comportamento sazonal e diário das abelhas Euglossina.

Na Mata do Pau-Ferro, a mudança no horário de atividade entre os períodos chuvosos e de estiagem pode ser devido às alterações na temperatura ambiental e umidade relativa observadas no período chuvoso, tendo em vista que o horário de atividade verificado no período de estiagem está de acordo com o normalmente observado para machos de Euglossina. Alocalização da mata, associada às variações sazonais na umidade, favorece as alterações na temperatura. As correlações significativas entre o número de indivíduos e a temperatura e umidade relativa observados no período chuvoso mostram que os valores de temperatura e umidade relativa situam-se próximos dos limiares necessários para o voo das abelhas. No período de estiagem, as condições climáticas estão acima dos valores limiares, não sendo, portanto, fatores limitantes.

As fragrâncias utilizadas neste estudo compõem a lista dos fortes atrativos para machos de Euglossina, conforme DressLER (1982). Em estudos realizados nas áreas de floresta atlântica do Brasil, eucaliptol (= cineol), eugenol e vanilina (= baunilha) foram utilizados em todos eles (REBÊLO \& GARÓFALO 1991, 1997; GARÓFALO et al. 1998; Peruquetti et al. 1999; Jesus \& Garófalo 2000; BEZERRA \& Martins 2001; BRITO \& RÊGO 2001; Silva \& REbÊLO 2002; Tonhasca et al. 2002b; Nemésio 2003; Sofia \& SUZUKI 2004; Sofia et al. 2004; Millet-Pinheiro \& Schlindwein 2005; Souza et al. 2005; Nemésio \& Silveira 2006; Farias et al. 2007, 2008; NemÉsio 2008; Nemésio 2010; Mattozo et al. 2011; Nemésio 2011). BezerRa \& Martins (2001) também utilizaram acetato de benzila, Souza et al. (2005) utilizaram as mesmas fragrâncias usadas neste estudo, excetuando o salicilato de metila, e MiLET-PinHEIro \& SCHLINDWEIN (2005) utilizaram exatamente as mesmas fragrâncias expostas na Mata do Pau-Ferro.

Eucaliptol tem sido a fragrância que atrai o maior número de espécies e indivíduos na maioria dos estudos realizados em áreas de floresta atlântica no Brasil (REBÊLo \& Garófalo 1991, 1997; Brito \& RêGo 2001; Silva \& RebÊlo 2002; Sofia \& Suzuki 2004; Sofia et al. 2004), excetuando Peruquetti et al. (1999), no qual o eugenol atraiu o maior número de espécies e vanilina o maior número de indivíduos, e TONHASCA et al. (2002b), NEMÉsIo (2003), Souza et al. (2005), Milet-Pinheiro \& Schlindwein (2005) e FARIAS et al. (2007), onde a beta ionona atraiu mais indivíduos. Nos três últimos casos isso provavelmente se deve, da mesma forma que neste estudo, ao fato de que a espécie mais abundante, E. carolina $(=E$. cordata), demonstrou preferência pela beta ionona. Caso essa fragrância não tivesse sido empregada, o eucaliptol provavelmente também teria sido responsável pela atração da maior parte dos indivíduos, já que o eucaliptol foi a segunda fragrância mais atrativa para E. carolina. Na Bahia, em todos os ecossistemas estudados eucaliptol foi a fragrância mais atrativa para espécies e indivíduos (NEvEs \& VIANA 2003).

O eugenol, o salicilato de metila e a vanilina também têm sido bons atrativos em várias áreas estudadas (REBÊLo \& GARÓFALO 1991, 1997; Peruquetti et al. 1999; Brito \& RÊGo 2001; Silva \& REBÊLO 2002; FARIAS et al. 2007). O acetato de benzila tem sido pouco atrativo quanto à abundância, mas foi responsável pela maioria das espécies atraídas nas áreas estudadas por BEZERRA \& Martins (2001) e FARIAS et al. (2007). O escatol tem sido pouco utilizado nos levantamentos feitos nas áreas de floresta atlântica no Brasil. Dentre aqueles autores que o utilizaram, somente NEMÉSIO (2003) e FARIAS et al. $(2007,2008)$ relataram as espécies atraídas. No primeiro caso, a fragrância atraiu três das sete espécies amostradas e, nos demais, quatro. Além disso, neste estudo, essa fragrância atraiu grande número de indivíduos de $E$. nigrita, espécie importante nos estudos de marcação e recaptura com fins a estimativas do tamanho populacional e determinação das distâncias de voo dessas abelhas.

Aoseanalisaremasfragrâncias visitadas pelas espéciesamostradas nas diversas áreas de floresta atlântica no Brasil, constata-se que apenas para algumas espécies é possível estabelecer aquelas que são as preferidas, devido ao baixo número de indivíduos coletados. Euglossa carolina ( $=$ E. cordata) tem mostrado preferência por beta ionona e eucaliptol (cineol) (REBÊLO \& GARÓFALO 1991; BEZERRA \& Martins 2001; Silva \& Rebêlo 2002; Farias et al. 2007), E. fimbriata tem preferido quase exclusivamente eucaliptol (REBÊLo \& Garófalo 1997; Peruquetti et al. 1999; Sofia et al. 2004), E. cingulata tem sido coletada na sua grande maioria no acetato de benzila, escatol e eugenol (Peruquetti et al. 1999; Bezerra \& 
Martins 2001; Brito \& RÊGo 2001; NemÉsIo 2003; FARIAS et al. 2007) e E. nigrita tem preferido escatol, eucaliptol e vanilina (Rebêlo \& Garófalo 1991, 1997; Peruquetti et al. 1999; Bezerra \& Martins 2001; Silva \& REBÊLO 2002; NemÉsio 2003; Sofia et al. 2004; FARIAS et al. 2007).

Segundo Oliveira \& CAmpos (1996), é muito difícil explicar a razão das preferências dos machos por determinadas fragrâncias. De acordo com Oliveira \& CAMpos (1995), apesar de muitas iscas terem sido utilizadas em diversos estudos na Amazônia, as abelhas parecem preferir um número reduzido delas, visitando pouco ou nada as demais. Das 16 fragrâncias utilizadas por PERUQUeTTI et al. (1999) em Minas Gerais, nove não foram atrativas. Por outro lado, embora as fragrâncias sejam a melhor ferramenta para se utilizar nos levantamentos faunísticos de Euglossina, alguns autores registraram que algumas espécies coletadas em flores não visitaram as iscas, como, por exemplo, Eufriesea auriceps Friese e Euglossa townsendi Cockerell (REBÊLO \& GARÓFALO 1991). Euglossa mandibularis Friese também não tem sido atraída por fragrâncias nos locais de sua ocorrência (PERUQuetTi et al. 1999).

Conquanto haja muitos trabalhos mostrando as fragrâncias preferidas pelas diversas espécies de Euglossina, ainda não há uma padronização com relação àquelas que trariam melhores resultados caso fossem empregadas. A variação nas fragrâncias que são utilizadas pode concorrer para resultados inverossímeis quanto às espécies que realmente são as mais abundantes.

Outrofenômeno comumenteobservado com relação às fragrâncias é a variação geográfica na preferência por essas substâncias (ACKERMAN 1989). RebêLo \& GARÓFALO (2001) citam o exemplo da espécie E. nigrita. Nas presenças de escatol e eucaliptol, machos dessa espécie têm mostrado preferência pela primeira no Panamá, em Ribeirão Preto e Santa Rosa do Viterbo, São Paulo; e pela segunda em Salvador, Bahia, como observado no presente estudo. O mesmo pode ser constatado para E. carolina quando comparadas as atratividades da beta ionona e do eucaliptol. Em oito coletas mensais no Parque Estadual do Rio Doce, Minas Gerais, PeruquetTi et al. (1999) utilizaram tanto a beta ionona quanto o eucaliptol e não coletaram nenhum indivíduo de $E$. carolina. Por outro lado, em dez coletas quinzenais realizadas em Viçosa os autores coletaram 132 machos de $E$. carolina no eucaliptol, mas não usaram a beta ionona. Nas áreas do nordeste onde a beta ionona e o eucaliptol foram utilizados, a espécie tem mostrado preferência pela primeira (Milet-PinheIro \& SCHLINDWEIN 2005; FARIAS et al. 2007).

O objetivo do uso das fragrâncias é estimar as densidades populacionais relativas das numerosas espécies que ocorrem numa determinada localidade (Powell \& Powell 1987). RoubiK (2004) ressaltou a importância de se entender a preferência dos machos de Euglossina por determinadas fragrâncias. O autor lembra que o número de espécies conhecidas só foi possível graças à utilização das fragrâncias artificiais. Mesmo assim, com o advento das fragrâncias na década de 1960, os estudos de levantamento só se intensificaram na década de 1990. Portanto, quanto mais estudos forem feitos, comparando o poder de atratividade das fragrâncias nas diferentes regiões do Brasil e também dos outros países no neotrópico, mais eficazes serão os levantamentos faunísticos de Euglossina e maior será o entendimento da relação dessas abelhas com as substâncias aromáticas.

\section{AGRADECIMENTOS}

Os autores são gratos a Antonio e Helena pelo acolhimento durante os períodos de coleta, aos amigos Maria Helena Peixoto e Paulo Ferreira pela ajuda nas coletas, à Empresa Viação Bela Vista pela concessão de passagens intermunicipais e à Coordenação de Aperfeiçoamento de Pessoal de Nível Superior (CAPES) pela bolsa concedida ao primeiro autor.

\section{REFERÊNCIAS}

Ackerman, J.D., 1983a. Diversity and seasonality of male euglossine bees (Hymenoptera: Apidae) in central Panama. Ecology, 64: 274-283.

Ackerman, J.D., 1983b. Specificity and mutual dependency of the orchid-euglossine bee interaction. Biological Journal of the Linnean Society, 20: 301-314.

Ackerman, J.D., 1989. Geographic and seasonal variation in fragrance choices and preferences of male euglossine bees. Biotropica, 21: 340-347.

Aguiar, W.M. \& M.C. Gaglianone. Euglossine bee communities in small forest fragments of the Atlantic Forest, Rio de Janeiro state, southeastern Brazil (Hymenoptera, Apidae). Revista Brasileira de Entomologia, 56: 210-219.

Almeida, L.M., C.S. Ribeiro-Costa, \& L. Marinoni, 2012. Coleta, conservação, montagem e identificação de insetos, p. 175190. In: Rafael, J.A., G.A.R. Melo, C. J.B.Carvalho, S.A. Casari \& R. Constantino (Eds.). Insetos do Brasil. Diversidade e taxonomia. Ribeirão Preto, Holos, 796p.

Ambruster, W.S. \& E.E. Berg, 1994. Thermal ecology of male euglossine bees in a tropical wet forest: fragrance foraging in relation to operative temperature. Biotropica, 26: 50-60.

Barbosa, M.R.V., M.F. Agra, E.V.S.B. Sampaio, J.P. Cunha \& L.A. Andrade, 2004. Diversidade florística da Mata do Pau-Ferro, Areia, Paraíba, p. 111-122. In: Pôrto, K.C., J.J.P. Cabral \& M. Tabarelli (Eds.). Brejos de altitude em Paraíba e Pernambuco - história natural, ecologia e conservação. Biodiversidade, Volume 9. Brasília, MMA/UFPE, 324p.

Becker, P., J.S. Moure \& F.J.A. Peralta, 1991. More about Euglossina bees in Amazonian forest fragments. Biotropica, 23: 586-591.

Bezerra, C.P. \& C.F. Martins, 2001. Diversidade de Euglossina (Hymenoptera, Apidae) em dois fragmentos de Mata Atlântica localizados na região urbana de João Pessoa, Paraíba, Brasil. Revista Brasileira de Zoologia, 18: 823-835.

Braga, P.I.S., 1976. Atração de abelhas polinizadoras de Orchidaceae com auxílio de iscas-odores na campina, campinarana e floresta tropical úmida da região de Manaus. Ciência e Cultura, 28: 767-773.

Brito, C.M.S. \& M.M.C. Rêgo, 2001. Community of male Euglossini bees (Hymenoptera: Apidae) in a secondary Forest, Alcântara, MA, Brazil. Brazilian Journal of Biology, 61: 631-638.

Carvalho, C.C., M.M.C. Rêgo \& F.N. Mendes, 2006. Dinâmica de populações de Euglossina (Hymenoptera, Apidae) em mata ciliar, Urbano Santos, Maranhão, Brasil. Iheringia Série Zoologia, 96: 249-256.

Cavalcanti, F.B., 2004. RPPN Fazenda Bituri. Disponível em <www.cprh.pe.gov.br/unidades conservacao/Uso Sustentavel/RPPN Fazenda Bituri/40043\%3B53640\%3B2 23909\%3B0\%3Bo.asp > [13/01/2013].

CONAMA - Conselho Nacional do Meio Ambiente, 1993. Resolução CONAMA n ${ }^{\circ}$ 10, de $1^{\circ}$ de outubro de 1993. Estabelece os parâmetros básicos para análise dos estágios de sucessão de Mata Atlântica. Disponível em <www.mma.gov. $\mathrm{br} /$ port/conama/legiabre.cfm?codlegi=135> [13/01/2013].

Dodson, C.H., R.L. Dressler, H.G. Hills, R.M. Adams \& N.H. Williams, 1969. Biologically active compounds in orchid fragrances. Science, 164: 1243-1249.

Dressler, R.L., 1982. Biology of the orchid bees (Euglossini). Annual Review of Ecology, Evolution, and Systematics, 13: 373-394.

Farias, R.C.A.P., M.C. Madeira-da-Silva, M.H. Pereira-Peixoto \& C.F. Martins, 2007. Horário de atividade de machos de Euglossina (Hymenoptera: Apidae) e preferência por fragrâncias artificiais em mata e dunas na Área de Proteção Ambiental da Barra do Rio Mamanguape, Rio Tinto, PB. Neotropical Entomology, 36: 863-867.

Farias, R.C.A.P., M.C. Madeira-da-Silva, M.H. Pereira-Peixoto \& C.F. Martins, 2008. Composição e sazonalidade de espécies de Euglossina (Hymenoptera: Apidae) em mata e duna na 
Área de Proteção Ambiental da Barra do Rio Mamanguape, Rio Tinto, PB. Neotropical Entomology, 37: 253-258.

Garófalo, C.A., 2006. Comportamento social nos Euglossini (Hymenoptera, Apidae), p. 10-13. In: Anais do VII Encontro sobre Abelhas. Ribeirão Preto.

Garófalo, C.A., E. Camillo, S.C. Augusto, B.M.V. Jesus \& J.C. Serrano, 1998. Diversidade e abundância sazonal de Euglossini (Hymenoptera, Apidae) na Serra do Japi, Jundiaí, SP, p. 72-79. In: Anais do IV Simpósio de Ecossistemas Brasileiros, volume 3. Águas de Lindóia.

Inouye, D.W., 1975. Flight temperatures of male euglossine bees (Hymenoptera: Apidae: Euglossini). Journal of the Kansas Entomological Society, 48: 366-370.

Janzen, D.H., P.J. Devries, M.L. Higgins \& L.S. Kimsey, 1982. Seasonal and site variation in Costa Rica euglossine bees at chemical baits in lowland deciduous and evergreen forests. Ecology, 63: 66-74.

Jesus, B.M.V. \& C.A. Garófalo, 2000. Riqueza e abundância sazonal de Euglossini (Hymenoptera, Apidae) na Mata da Virgínia, Matão, São Paulo, p 239-245. In: Anais do IV Encontro sobre Abelhas. Ribeirão Preto.

Mattozo, V.C., L.R.R. Faria \& G.A.R. Melo, 2011. Orchid bees (Hymenoptera: Apidae) in the coastal forests of southern Brazil: diversity, efficiency of sampling methods and comparison with other Atlantic forest surveys. Papéis Avulsos de Zoologia, 51: 505-515.

Mayo, S.J. \& V.P.B. Fevereiro, 1982. Mata do Pau-Ferro: a pilot study of the brejo forest of Paraiba, Brazil. London, Royal Botanic Gardens, 29p.

Michener, C.D., 200o. The bees of the world. Baltimore, The Johns Hopkins University Press, 913p.

Milet-Pinheiro, P. \& C. Schlindwein, 2005. Do euglossine males (Apidae, Euglossini) leave tropical rainforest to collect fragrances in sugarcane monocultures? Revista Brasileira de Zoologia, 22: 853-858.

MMA- Ministério do Meio Ambiente, 2002. Avaliação e identificação de áreas e ações prioritárias para conservação, utilização sustentável e repartição de benefícios da biodiversidade brasileira. Brasília, MMA/SBF, 404p.

Moure, J.S., G.A.R. Melo \& L.R.R. Faria, 2007. Euglossini Latreille, 1802, p. 214-255. In: Moure, J.S., D. Urban \& G.A.R. Melo (Eds.). Catalogue of bees (Hymenoptera, Apoidea) in the Neotropical Region. Curitiba, Sociedade Brasileira de Entomologia, 1058p.

Nemésio, A. F. \& Silveira, 2006. Edge effects on the orchid-bee fauna (Hymenoptera, Apidae) at a large remnant of Atlantic rain forest in southeastern Brazil. Neotropical Entomology, 35: $313-323$.

Nemésio, A., 2003. Preliminary sampling of Euglossina (Hymenoptera: Apidae: Apini) of Reserva Particular do Patrimônio Natural Feliciano Miguel Abdala, Caratinga, Minas Gerais, southeastern Brazil. Lundiana 4: 121-124.

Nemésio, A., 2008. Orchid bee community (Hymenoptera, Apidae) at an altitudinal gradient in a large forest fragment in southeastern Brazil. Revista Brasileira de Zoociências, 10: 249-256.

Nemésio, A., 2010. The orchid-bee fauna (Hymenoptera: Apidae) of a forest remnant in northeastern Brazil, with new geographic records and an identification key to the known species of the Atlantic forest of northeastern Brazil. Zootaxa, 2656: $55-66$

Nemésio, A., 2011. The orchid-bee fauna (Hymenoptera: Apidae) of a forest remnant in southern Bahia, Brazil, with new geographic records and an identification key to the known species of the area. Zootaxa, 2821: 47-54

Neves, E.L. \& B.F. Viana, 1997. Inventário da fauna de Euglossina (Hymenoptera, Apidae) do baixo sul da Bahia, Brasil. Revista Brasileira de Zoologia, 14: 831-837.

Neves, E.L. \& B.F. Viana, 1999. Comunidade de machos de Euglossina (Hymenoptera, Apidae) das matas ciliares da margem esquerda do médio rio São Francisco, Bahia. Anais da Sociedade Entomológica do Brasil, 28: 201-210.

Neves, E.L. \& B.F. Viana, 2003. A fauna de abelhas da subtribo Euglossina (Hymenoptera, Apidae) do estado da Bahia, Brasil, p. 223-229. In: Melo, G.A.R. \& I. Alves-dos-Santos (orgs.). Apoidea Neotropica: Homenagem aos 90 Anos de Jesus Santiago Moure. Criciúma, UNESC, 320p.

Oliveira, M.L. \& L.A.O. Campos, 1995. Abundância, riqueza e diversidade de abelhas Euglossina (Hymenoptera, Apidae) em florestas contínuas de terra firme na Amazônia Central, Brasil. Revista Brasileira de Zoologia, 12: 547-556.

Oliveira, M.L. \& L.A.O. Campos, 1996. Preferência por estratos florestais e por substâncias odoríferas em abelhas Euglossina (Hymenoptera, Apidae). Revista Brasileira de Zoologia, 13: 1075-1085.

Oliveira, M.L., 1999. Sazonalidade e horário de atividade de abelhas Euglossina (Hymenoptera, Apidae) em florestas de terra firme na Amazônia Central. Revista Brasileira de Zoologia, 16: 83-90.

Peruquetti, R.C., L.A.O. Campos, C.D.P. Coelho, C.V.M. Abrantes \& L.C.V. Lisboa, 1999. Abelhas Euglossini (Apidae) de áreas de mata atlântica: abundância, riqueza e aspectos biológicos. Revista Brasileira de Zoologia, 16: 101-118.

Pôrto, K.C., J.J.P. Cabral \& M. Tabarelli, 2004. Brejos de altitude em Paraíba e Pernambuco - história natural, ecologia e conservação. Biodiversidade, Volume 9. Brasília, MMA/ UFPE, 324p.

Powell, A. H. \& G.V.N. Powell, 1987. Population dynamics of male euglossine bees in Amazonian forest fragments. Biotropica, 19: $176-179$

Rebêlo, J.M.M. \& A.J.M. Cabral, 1997. Abelhas Euglossinae de Barreirinhas, zona do litoral da Baixada Oriental Maranhense. Acta Amazônica, 27: 145-152.

Rebêlo, J.M.M. \& C.A. Garófalo, 1991. Diversidade e sazonalidade de machos de Euglossini (Hymenoptera, Apidae) e preferências por iscas-odores em um fragmento de floresta no sudeste do Brasil. Revista Brasileira de Biologia, 51: 787799.

Rebêlo, J.M.M. \& C.A. Garófalo, 1997. Comunidades de machos de Euglossini (Hymenoptera: Apidae) em matas semidecíduas do nordeste do estado de São Paulo. Anais da Sociedade Entomológica do Brasil, 26: 243-255.

Rebêlo, J.M.M., 2001. História natural das euglossíneas - as abelhas das orquídeas. São Luís, Lithograf, 152p.

Roubik, D.W \& P.E. Hanson, 2004. Abejas de orquídeas de la América tropical. Biología y guía de campo. Costa Rica, INBio, 370p.

Roubik, D.W. \& J.D. Ackerman, 1987. Long-term ecology of euglossine orchid-bees (Apidae: Euglossini) in Panama. Oecologia, 73: 321-333.

Roubik, D.W., 2001. Ups and downs in pollinator populations: when is there a decline? Conservation Ecology, 5: 1-22.

Roubik, D.W., 2004. Long-term studies of solitary bees: What the orchid bees are telling us, p. 97-103. In: Freitas, B.M. \& J.O.P. Pereira (orgs.). Solitary bees. Conservation, rearing and management for pollination. Fortaleza, Imprensa Universitária, 285p.

Santos, A.M. \& S.H. Sofia, 2002. Horário de atividade de machos de Euglossinae (Hymenoptera, Apidae) em um fragmento de floresta semidecídua no norte do estado do Paraná. Acta Scientiarum, 24: 375-381.

Silva, F.S. \& J.M.M. Rebêlo, 1999. Euglossine bees (Hymenoptera: Apidae) of Buriticupu, Amazonia of Maranhão, Brazil. Acta Amazonica, 29: 587-599.

Silva, F.S. \& J.M.M. Rebêlo, 2002. Population dynamics of Euglossinae bees (Hymenoptera, Apidae) in an early secondgrowth forest of Cajual Island, in the state of Maranhão, Brazil. Brazilian Journal of Biology, 62: 15-23.

Silveira, F.A., G.A.R. Melo \& E.A.B. Almeida, 2002. Abelhas brasileiras - sistemática e identificação. Belo Horizonte, F.A. Silveira, 253p.

Sofia, S.H. \& K.M. Suzuki, 2004. Comunidades de machos de 
abelhas euglossina (Hymenoptera: Apidae) em fragmentos florestais no sul do Brasil. Neotropical Entomology, 33: 693702.

Sofia, S.H., A.M. Santos \& C.R.M. Silva, 2004. Euglossine bees (Hymenoptera, Apidae) in a remnant of Atlantic Forest in Paraná State, Brazil. Iheringia Série Zoologia, 94: 217-222.

Souza, A.K.P., M.I.M Hernández \& C.F. Martins, 2005. Riqueza, abundância e diversidade de Euglossina (Hymenoptera, Apidae) em três áreas da Reserva Biológica Guaribas, Paraíba, Brasil. Revista Brasileira de Zoologia, 22: 320-325.

Tabarelli, M. \& A.M.M. Santos, 2004. Uma breve descrição sobre a história natural dos brejos nordestinos, p. 17-24. In: Pôrto, K. C., J. J. P. Cabral \& M. Tabarelli (Eds.). Brejos de altitude em Paraíba e Pernambuco - história natural, ecologia e conservação. Biodiversidade, Volume 9. Brasília, MMA/ UFPE, 324p.

Tonhasca, A., G.S. Albuquerque \& J. L. Blackmer, 2002a. Dispersal of euglossine bees between fragments of the Brazilian Atlantic Forest. Journal of Tropical Ecology, 19: 99-102.
Tonhasca, A., J. L. Blackmer \& G.S. Albuquerque, 2002b. Abundance na diversity of euglossine bees in the fragmented landscape of the Brazilian Atlantic forest. Biotropica, 34: 416422.

Vasconcelos-Sobrinho, J., 1971. As regiões naturais do nordeste, o meio e a civilização. Recife, Conselho de Desenvolvimento de Pernambuco, 441p.

Viana, B.F., A.M.P. Kleinert \& E.L. Neves, 2002. Comunidade de Euglossini (Hymenoptera, Apidae) das dunas litorâneas do Abaeté, Salvador, Bahia, Brasil. Revista Brasileira de Entomologia, 46: 539-545.

Williams, N.H. \& W.M. Whitten, 1983. Orchid floral fragances and male euglossine bees: methods and advances in last sesquidecade. The Biological Bulletin, 164: 355-395.

\section{Recebido em: 15/o1/2013 \\ Aceito em: 09/07/2013}

\section{Como citar este artigo:}

Farias, R.C.A.P. \& C.F. Martins, 2013. Sazonalidade e Padrões Diários de Atividade de Machos de Euglossina (Hymenoptera: Apidae: Apini) e Preferências por Fragrâncias Artificiais em um Remanescente de Brejo de Altitude na Paraíba. EntomoBrasilis, 6(3): 202-209.

Acessível em: http://www.periodico.ebras.bio.br/ojs/index.php/ebras/article/view/312. doi:10.12741/ebrasilis.v6i3.312 